\title{
The Worst-Case Interference in DSL Systems Employing Dynamic Spectrum Management
}

\author{
Mark H. Brady and John M. Cioffi \\ Department of Electrical Engineering, Stanford University, Stanford, CA 94305-9515, USA
}

Received 1 December 2004; Revised 28 July 2005; Accepted 31 July 2005

\begin{abstract}
Dynamic spectrum management (DSM) has been proposed to achieve next-generation rates on digital subscriber lines (DSL). Because the copper twisted-pair plant is an interference-constrained environment, the multiuser performance and spectral compatibility of DSM schemes are of primary concern in such systems. While the analysis of multiuser interference has been standardized for current static spectrum-management (SSM) techniques, at present no corresponding standard DSM analysis has been established. This paper examines a multiuser spectrum-allocation problem and formulates a lower bound to the achievable rate of a DSL modem that is tight in the presence of the worst-case interference. A game-theoretic analysis shows that the rate-maximizing strategy under the worst-case interference (WCI) in the DSM setting corresponds to a Nash equilibrium in pure strategies of a certain strictly competitive game. A Nash equilibrium is shown to exist under very mild conditions, and the rate-adaptive waterfilling algorithm is demonstrated to give the optimal strategy in response to the WCI under a frequency-division (FDM) condition. Numerical results are presented for two important scenarios: an upstream VDSL deployment exhibiting the near-far effect, and an ADSL RT deployment with long CO lines. The results show that the performance improvement of DSM over SSM techniques in these channels can be preserved by appropriate distributed power control, even in worst-case interference environments.
\end{abstract}

Copyright (c) 2006 M. H. Brady and J. M. Cioffi. This is an open access article distributed under the Creative Commons Attribution License, which permits unrestricted use, distribution, and reproduction in any medium, provided the original work is properly cited.

\section{INTRODUCTION}

In recent years, increased demands on data rates and competition from other services have led to the development of new high-speed transmission standards for digital subscriber line (DSL) modems. Dynamic spectrum management (DSM) is emerging as a key component in next-generation DSL standards. In DSM, spectrum is allocated adaptively in response to channel and interference conditions, allowing mitigation of interference and best use of the channel. As multiuser interference is the primary limiting factor to DSL performance, the potential for rate improvement by exploiting its structure is substantial.

DSM contrasts with current DSL practice, known as static spectrum management (SSM). In SSM, masks are imposed on transmit power spectrum densities (PSDs) to bound the amount of crosstalk induced in other lines sharing the same binder group [1]. As SSM masks are fixed for all loop configurations, they can often be far from optimal or even prudent spectrum usage in typical deployments. Standardized tests for "spectral compatibility" [1] assess "new technology" by defining PSD masks and examining the impact on standardized systems using the 99th-percentile cross- talk scenario. Such methods are useful when a reasonable estimate of spectrum of all users can be assumed priori. However, if spectrum is instead allocated dynamically, not only is this knowledge not available priori, but also because of loop unbundling, other users' spectrum may not even be known even during operation. Spectral compatibility between different operators using DSM is a primary concern because new pathologies may arise with adaptive operation. Moreover, it is not unreasonable to suspect that each competing service provider sharing a binder would perform DSM in a greedy fashion, at the possible expense of other providers' users. However, in DSM, a worst-case interference analysis based on maximum allowable PSDs is overly pessimistic, so existing spectral compatibility techniques cannot be fruitfully employed. A new paradigm is needed to assess the impact of DSM on multiuser performance of the overall system.

\subsection{Prior results}

The capacity region of the AWGN interference channel (IC) is in general unknown, even for the 2-user case [2]. Communication in the presence of hostile interference has been studied from a game-theoretic perspective in numerous 


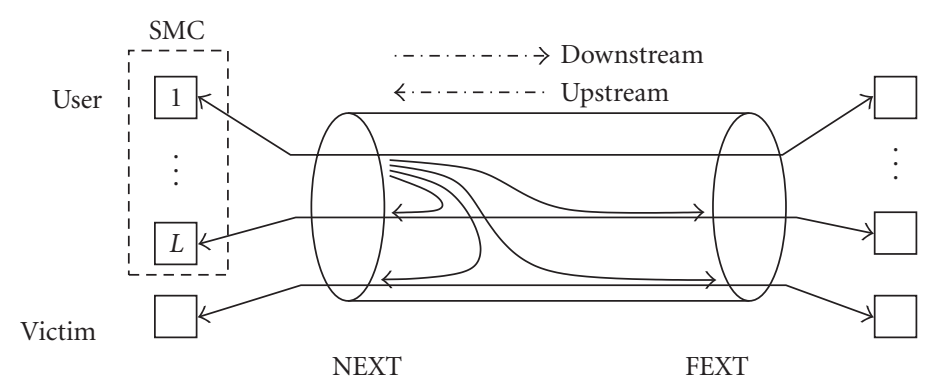

FIGURE 1: Illustration of loop plant environment showing downstream FEXT and NEXT from user 1. The victim user is shown at the bottom.

applications, for example, $[3,4]$. A simple and relevant IC achievable region is that attained by treating interference as noise [5]. Capacity results for frequency-selective interference channels satisfying the strong interference condition are also known [6].

DSM algorithms have been proposed for the cases of distributed and centralized control scenarios. This paper considers what has been termed "Level 0-2 DSM" [7], wherein cooperation may be allowed to manage spectrum, but not for multiuser encoding and decoding. A centralized DSM center controlling multiple lines offers both higher potential performance and improved management capabilities [8]. Distributed DSM schemes based on the iterative waterfilling (IW) algorithm [9] have been presented. IW has also been studied from a game-theoretic viewpoint [10]. Numerous algorithms for centralized DSM have been proposed. Reference [11] presents a technique to maximize users' weighted sumrate. Rate maximization subject to frequency-division and fixed-rate proportions between users has been considered [12]. Optimal [13] and suboptimal [14] algorithms to minimize transmit power have been studied.

An extensive suite of literature on upstream powerbackoff techniques to mitigate the "near-far" problem has been developed for static spectrum-management systems [13, 15-17]. A power-backoff algorithm for DSM systems implementing iterative waterfilling has been proposed [18].

In current DSL standards, upstream and downstream transmissions use either distinct frequency bands or shared bands. In the latter case, "echo" is created between upstream and downstream transmissions [9]. As analog hybrid circuits do not provide sufficient isolation, echo mitigation is essential in practical systems [19]. Numerous echo-cancellation structures have been proposed for DSL transceivers [20-22].

\subsection{Outline}

This paper formulates the achievable rate of a single "victim" modem in the presence of the worst-case interference from other interfering lines in the same binder group. The performance under the WCI is a guaranteed-achievable rate that can be used, for example, in studying multiuser performance of DSM strategies and establishing spectral compatibility of DSM systems.

Section 2 defines the channel and system models. The WCI problem is formalized and studied in Section 3 from a game-theoretic viewpoint. Certain properties of the Nash equilibrium of this game are explored. Section 4 considers numerical examples in VDSL and ADSL systems. Concluding remarks are made in Section 5.

A word on notation: vectors are written in boldface, where $\mathbf{v}_{k}$ denotes the $k$ th element of the vector $\mathbf{v}$, and $\mathbf{v} \geq 0$ denotes that each element is nonnegative. The notation $\mathbf{v}^{(n)}$ denotes a vector corresponding to tone $n$. For the symmetric matrix $X, X \geq 0$ denotes that $X$ is positive semidefinite. 1 is a column vector with each element equal to 1 . $\operatorname{int}(X)$ denotes the (topological) interior, $\operatorname{cl}(X)$ the closure, and $\partial X$ the boundary of the set $X$.

\section{SYSTEM MODEL}

\subsection{Channel model}

A copper twisted-pair DSL binder is modelled as a frequencyselective multiuser Gaussian interference channel $[9,23]$. The binder contains a total of $L+1$ twisted pairs, with one DSL line per twisted pair, as shown in Figure 1. The effect of NEXT and FEXT interferences generated by $L$ "interfering" users that generate crosstalk into one "victim" user is considered. This coupling is illustrated for downstream transmission in Figure 1.

\subsection{DSL modem model}

\subsubsection{Modem architecture}

The standardized [24] discrete-multitone (DMT)-based modulation scheme is employed, so that transmission over the frequency-selective channel may be decoupled into $\mathrm{N}$ independent subcarriers or tones. Both FDM and overlapping bandplans are considered. As overlapping bandplans require echo cancellation that is imperfect in practice, error that is introduced acts as a form of interference and is of concern. Echo-cancellation error is modelled presuming a prevalent echo-cancellation structure utilizing a joint time-frequency LMS algorithm [19] is employed. ${ }^{1}$ Using the terminology of [19], let $\mu$ denote the LMS adaptive step size parameter. The "excess MSE" for a given tone is modelled [25, equation

\footnotetext{
${ }^{1}$ Other models may be more applicable to different echo-cancellation structures.
} 
(12.74)] as proportional to the product of the LMS adaptive step size parameter $\mu$ and the transmit power on that tone. The constant of proportionality is absorbed by defining $\hat{\beta}$ as the ratio of excess MSE to transmitted energy on a given tone.

\subsubsection{Achievable rate region}

This section discusses an achievable rate region for a DSL modem based on the preceding channel and system model. The following analysis applies to both upstream and downstream transmissions. For specificity, the following refers to downstream transmission: first, consider the case where echo cancellation is employed. Denote the victim modem's downstream transmit power on tone $n, n \in\{1, \ldots, N\}$, as $\mathbf{x}_{n}$. Let element $l, l \in\{1, \ldots, L\}$, of the vector $\mathbf{y}^{(n)} \in \mathbb{R}_{+}^{2 L}$ denote the downstream transmit power of interfering modem $l$ on tone $n$. Similarly, let element $l, l \in\{L+1, \ldots, 2 L\}$, of $\mathbf{y}^{(n)}$ denote the upstream transmit power of interfering user $l-L$. Define element $l, l \in\{l, \ldots, L\}$, of the row vector $\mathbf{h}^{(n)} \in \mathbb{R}_{+}^{2 L}$ as the FEXT power gain from interfering user $l$ on tone $n$ (necessarily, $\left.\mathbf{h}^{(n)} \succeq 0\right)$. Similarly, define element $l, l \in\{L+1, \ldots, 2 L\}$, of $\mathbf{h}^{(n)}$ to be the NEXT power gain from interfering user $l-L$. Let element $n$ of $\widetilde{\mathbf{h}}_{n} \in \mathbb{R}_{+}^{N}$ denote the victim line's insertion gain on tone $n\left(\tilde{\mathbf{h}}_{n} \geq 0\right)$.

Independent AWGN (thermal noise) with power $\sigma_{n}^{2}>0$ is present on tone $n$. Let $\widehat{\beta_{n}}$ denote the echo-cancellation ratio on tone $n$ as described above. Echo-cancellation error is treated as AWGN. Let $\Gamma$ denote the SNR gap-to-capacity [9]. Then the following bit loading ${ }^{2}$ is achievable on tone $n$ [9]:

$$
b_{n}=\log \left(1+\frac{\tilde{\mathbf{h}}_{n} \mathbf{x}_{n}}{\Gamma\left(\mathbf{h}^{(n)} \mathbf{y}^{(n)}+\hat{\beta} \mathbf{x}_{n}+\sigma_{n}^{2}\right)}\right) .
$$

Observe that if $\tilde{\mathbf{h}}_{n}=0$, then it is necessarily the case that $b_{n}=0$, implying that tone $n$ is never loaded. Thus, in the sequel, $\tilde{\mathbf{h}}_{n}>0$ for all $n \in\{1, \ldots, N\}$ is considered without loss of generality by removing those tones with zero direct gain $\left(\tilde{\mathbf{h}}_{n}=0\right)$. Defining $\alpha_{n}=\Gamma / \widetilde{\mathbf{h}}_{n}, \beta_{n}=\Gamma \widehat{\beta_{n}} / \tilde{\mathbf{h}}_{n}$, and $\mathbf{N}_{n}=$ $\Gamma \sigma_{n}^{2} / \tilde{\mathbf{h}}_{n}$, and substituting

$$
b_{n}=\log \left(1+\frac{\mathbf{x}_{n}}{\alpha_{n} \mathbf{h}^{(n)} \mathbf{y}^{(n)}+\beta_{n} \mathbf{x}_{n}+\mathbf{N}_{n}}\right) \text {, }
$$

because $\Gamma \geq 1$, it follows that $\alpha_{n} \geq 0, \beta_{n} \geq 0$, and $\mathbf{N}_{n}>0$.

\subsubsection{Achievable rate region for FDM}

When an FDM scheme is employed, NEXT and echo cancellation are eliminated because transmission and reception occur on distinct frequencies. ${ }^{3}$ As a common configuration

\footnotetext{
2 The achieved data rate of a given modem is proportional to the number of bits loaded (less overhead); this constant of proportionality is normalized to 1 in the theoretical development.

${ }^{3}$ Effects arising from implementation issues that may lead to crosstalk between upstream and downstream bands are not explicitly considered.
}

in ADSL and VDSL standards [9], this represents the important special case of the preceding model, where $\beta_{n}=0$ (due to no echo cancellation) and $\mathbf{h}_{l}^{(n)}=0$ for all $n, L+1 \leq l \leq 2 L$ (due to frequency division). Additional technical results will be shown to hold in the FDM setting, as detailed in Section 3.

\section{THE WORST-CASE INTERFERENCE}

\subsection{Game-theoretic characterization of the WCI}

This section introduces and motivates the concept of the worst-case interference (WCI). Suppose that a "victim" modem desires to keep its data rate at some level. Such a scenario is commonplace as carriers widely offer DSL service at fixed data rates. The objective is to bound the impact that multiuser interference can have on this victim modem, thereby determining whether service may be guaranteed. To this end, one considers interferences that are the most harmful in the sense of minimizing the achievable rate of a "victim" modem. However, it is not clear what form such interferences might take, nor how they might be best responded to.

Examining this problem from the standpoint of game theory leads to substantial insight. Consider a worst-case interference game where one player jointly optimizes the spectrum of all the interfering modems, irrespective of the data rate they achieve in doing so, to cause the most deleterious interference to the victim modem. Thus in this game, all the interfering modems act as one player, while the victim modem acts as the other player, with the channel and noise known to all. Although such an arrangement may appear pathological, it will be shown numerically that such a situation is quite close to what occurs in certain loop topologies. Neither is assuming such coordination of the interferers unreasonable in practice as under "Level 2" DSM [7, 8], each collocated carrier may individually coordinate its own lines, nor may collocated equipment be centrally controlled by a competing carrier. Channels may be estimated in the field, approximated by standardized models [9], and in the future, potentially published by operators [26].

A Nash equilibrium in this game may be interpreted as characterizing a worst-case interference as an optimal response (power-allocation policy) to it. The structure of the Nash equilibrium lends insight into the problem as well as suggesting techniques that may be implemented in practical systems.

\subsection{Formalization of the WCI game}

Consider the following two-player game: let Player 1 control the spectrum allocation of victim modem, and let Player 2 control the spectrum allocations of all the interfering modems. Referring again to downstream transmission for specificity, let the total (sum) downstream power of the victim modem $\sum_{n} \mathbf{x}_{\mathbf{n}}$ be upper bounded by $P^{x}$, where $0<P^{x}<$ $\infty$. Player 1 is also subject to a positive power constraint $\mathbf{C}^{x}$ on each tone, so that $\mathbf{x} \preceq \mathbf{C}^{x}$. Note that this constraint may be made redundant by setting, for example, $\mathbf{C}^{x} \succeq 1 P^{x}$. The requirement that $\mathbf{C}^{x}>0$ is without loss of generality by 
disregarding all unusable tones $n$ for which $\mathbf{C}_{n}^{x}=0$. Similarly for Player 2, consider per-line power constraints $0 \prec \mathbf{P}^{y} \prec \infty$, where the total downstream power of the $l$ th interfering modem $l \in\{1, \ldots, L\}$ is upper bounded by the $l$ th element of $\mathbf{P}^{y} \in \mathbb{R}_{++}^{2 L}$ and the total upstream power of interfering modem $l$ is upper bounded by element $l+L$ of $\mathbf{P}^{y}$. Further, consider positive power constraints $\mathbf{C}^{y,(n)} \in \mathbb{R}_{++}^{2 L}$ for $n=1, \ldots, N$ such that $\mathbf{y}^{(n)} \preceq \mathbf{C}^{y,(n)}$ for each $n$; any such power constraints equal to zero may be equivalently enforced by zeroing respective element(s) of $\left\{\mathbf{h}^{(n)}\right\}$.

The strategy set of Player 1 is the set of all feasible power allocations for the victim modem, $\varsigma_{1}=\{\mathbf{x}: 0 \preceq$ $\left.\mathbf{x} \preceq \mathbf{C}^{x}, \mathbf{1}^{T} \mathbf{x} \leq P^{x}\right\}$, and the strategy set of Player 2 is the set of all feasible power allocations for the interfering modems, $\wp_{2}=\left\{\left[\mathbf{y}^{(1)}, \ldots, \mathbf{y}^{(N)}\right]: 0 \preceq \mathbf{y}^{(n)} \preceq \mathbf{C}^{y,(n)}, n=\right.$ $\left.1, \ldots, N,\left[\mathbf{y}^{(1)}, \ldots, \mathbf{y}^{(N)}\right] \mathbf{1} \preceq \mathbf{P}^{y}\right\}$. Define $s=s_{1} \times s_{2}$. This is a strictly competitive or zero sum two-player game $\left(\wp_{1}, \S_{2}, J\right)$, where the objective function $J: \& \mapsto \mathbb{R}_{+}$is defined to be the achievable data rate of the victim user:

$J\left(\mathbf{x},\left[\mathbf{y}^{(1)}, \ldots, \mathbf{y}^{(N)}\right]\right)=\sum_{n=1}^{N} \log \left(1+\frac{\mathbf{x}_{n}}{\alpha_{n} \mathbf{h}^{(n)} \mathbf{y}^{(n)}+\beta_{n} \mathbf{x}_{n}+\mathbf{N}_{n}}\right)$.

The game $g=\left(\delta_{1}, s_{2}, J\right)$ is defined to be the worst-case interference game.

\subsection{Derivation of Nash equilibrium conditions}

A Nash equilibrium in pure strategies in the WCI game $g$ is defined to be any saddle point $\left(\mathbf{x},\left[\mathbf{y}^{(1)}, \ldots, \mathbf{y}^{(N)}\right]\right) \in \&$ satisfying

$$
\begin{aligned}
J\left(\tilde{\mathbf{x}},\left[\mathbf{y}^{(1)}, \ldots, \mathbf{y}^{(N)}\right]\right) & \leq J\left(\mathbf{x},\left[\mathbf{y}^{(1)}, \ldots, \mathbf{y}^{(N)}\right]\right) \\
& \leq J\left(\mathbf{x},\left[\tilde{\mathbf{y}}^{(1)}, \ldots, \tilde{\mathbf{y}}^{(N)}\right]\right),
\end{aligned}
$$

for all $\tilde{\mathbf{x}} \in \wp_{1},\left[\tilde{\mathbf{y}}^{(1)}, \ldots, \tilde{\mathbf{y}}^{(N)}\right] \in \oiint_{2}$. Condition (5) immediately implies the claim that Player 1 rate at a Nash equilibrium of $g$ lower bounds the achievable rate with any other feasible interference profile. This bound also extends to other settings: in the noncooperative IW game [10], a (possibly non-unique) Nash equilibrium is known to always exist in pure strategies; condition (5) again yields a lower bound rate at every Nash equilibrium of the IW game for the line corresponding to Player 1.

It is now shown that a Nash equilibrium of $g$ always exists due to certain properties of the objective and strategy sets. First, the convex-concave structure of the objective is established.

Theorem 1. If $\alpha \geq 0, \beta \geq 0, \gamma>0, \mathbf{h} \in \mathbb{R}_{+}^{2 L}$, and $\alpha, \beta, \gamma$, h are bounded, then the function $g: \mathbb{R}_{+} \times \mathbb{R}_{+}^{2 L} \mapsto \mathbb{R}_{+}$defined by

$$
g(x, \mathbf{y})=\log \left(1+\frac{x}{\alpha \mathbf{h}^{T} \mathbf{y}+\beta x+\gamma}\right)
$$

is strictly concave in $x$ and is convex in $\mathbf{y}$.

Proof. It is first shown that $f: \mathbb{R}_{+} \times \mathbb{R}_{+} \rightarrow \mathbb{R}_{+}, f(x, \eta)=$ $\log ((1+\beta) x+\alpha \eta+\gamma)-\log (\alpha \eta+\beta x+\gamma)$ is convex in $\eta$ and strictly concave in $x$. It is sufficient [27] to show that for all $x \geq 0$, it holds that $\partial^{2} f / \partial \eta^{2} \geq 0$ on the interval $(-\epsilon, \infty)$ for some $\epsilon>0$, and similarly for all $\eta \geq 0$ that $\partial^{2} f / \partial x^{2}<0$ on the interval $(-\epsilon, \infty)$ for some $\epsilon>0$. By differentiating and simplifying,

$$
\begin{gathered}
\frac{\partial f}{\partial x}=\frac{\alpha \eta+\gamma}{(\alpha \eta+\beta x+\gamma)((\beta+1) x+\alpha \eta+\gamma)} \\
\frac{\partial^{2} f}{\partial x^{2}}=\frac{(\alpha \eta+\gamma)(2 \beta(\beta+1) x+(2 \beta+1)(\alpha \eta+\gamma))}{-(\alpha \eta+\beta x+\gamma)^{2}(\alpha \eta+(\beta+1) x+\gamma)^{2}}<0 \\
\frac{\partial f}{\partial \eta}=-\frac{\alpha x}{(\alpha \eta+\beta x+\gamma)(\alpha \eta+(\beta+1) x+\gamma)} \\
\frac{\partial^{2} f}{\partial \eta^{2}}=\frac{\alpha^{2}(2 \alpha \eta+(2 \beta+1) x+2 \gamma) x}{(\alpha \eta+\beta x+\gamma)^{2}(\alpha \eta+(\beta+1) x+\gamma)^{2}} \geq 0
\end{gathered}
$$

where $\epsilon=\gamma /(4 \beta(\beta+1))$ in $(8), \epsilon=\gamma /(2 \alpha)$ when $\alpha>0$, and $\epsilon=1$ when $\alpha=0$ in (10). For all $(x, y) \in \mathbb{R}_{+} \times \mathbb{R}_{+}^{2 L}$, it must be that $\mathbf{h}^{T} \mathbf{y} \geq 0$. Thus $g(x, \mathbf{y})=f\left(x, \mathbf{h}^{T} \mathbf{y}\right)$. By the affine mapping composition property [27], it follows that $g(x, y)$ is convex in $\mathrm{y}$ and strictly concave in $x$.

Because the objective (3) is a sum of functions that are strictly concave in $\mathbf{x}_{\mathbf{n}}$ and convex in $\mathbf{y}^{(n)}, J$ is strictly concave in $\mathbf{x}$ and convex in $\left[\mathbf{y}^{(1)}, \ldots, \mathbf{y}^{(N)}\right]$.

Theorem 2. The WCI game g has a Nash equilibrium existing in pure strategies, and a value $R^{*}$.

Proof. Because $\ell_{1} \subset \mathbb{R}^{N}$ and $\ell_{2} \subset \mathbb{R}^{2 L N}$ are closed and bounded, by the Heine-Borel theorem, they are both compact. Also, the objective is a composition of continuous functions, hence continuous, and $J$ is strictly concave in $\mathbf{x}$ and convex in $\left[\mathbf{y}^{(1)}, \ldots, \mathbf{y}^{(N)}\right]$. The conditions of $[28$, Theorem 4.4] are thus satisfied, and therefore a pure-strategy saddle point exists. Note that the saddle point need not be unique, in general. Because a saddle point exists in pure strategies, the game has a value [28, Theorem 4.1], which will be denoted as $R^{*}$. Thus,

$$
\max _{\mathbf{x} \in S_{1}} \min _{\left[\mathbf{y}^{(1)}, \ldots, \mathbf{y}^{(N)}\right] \in \mathcal{S}_{2}} J=\min _{\left[\mathbf{y}^{(1)}, \ldots, \mathbf{y}^{(N)}\right] \in S_{2}} \max _{\mathbf{x} \in S_{1}} J=R^{*}
$$

\subsection{Structure of the worst-case interference}

The previous section showed that under very general conditions, a Nash equilibrium exists. However, it is not immediately clear whether there exists a unique Nash equilibrium, or whether Nash equilibria of the WCI game might possess any simplifying structure.

The former question may be addressed by considering the following example: $N=2, L=2, \mathbf{h}^{(1)}=\mathbf{h}^{(2)}=$ $\left[\begin{array}{llll}1 & 1 & 0 & 0\end{array}\right], P^{x}=1, \mathbf{P}^{y}=\left[\begin{array}{ll}1 & 1\end{array}\right]^{T}, \mathbf{N}_{1}=\mathbf{N}_{2}>0, \alpha_{1}=\alpha_{2}=1$, $\Gamma=1$, and suppose that the FDM condition is satisfied and the per-tone power constraints are redundant. Then it may be readily verified by symmetry arguments that with $\mathbf{x}=$ $\left[\begin{array}{ll}1 / 2 & 1 / 2\end{array}\right]^{T}$, both $\mathbf{y}^{(1)}=\left[\begin{array}{llll}1 & 0 & 0 & 0\end{array}\right]^{T}, \mathbf{y}^{(2)}=\left[\begin{array}{llll}0 & 1 & 0 & 0\end{array}\right]^{T}$ 
and $\mathbf{y}^{(1)}=\mathbf{y}^{(2)}=\left[\begin{array}{llll}1 / 2 & 1 / 2 & 0 & 0\end{array}\right]^{T}$ (and convex combina-

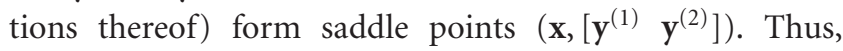
Player 2 may have an uncountably infinite number of optimal strategies even under the FDM condition, and hence the saddle point need not to be unique in general.

Given that the Nash equilibrium is not generally unique, its structure is explored in the following results. Some basic intuition is first established showing that "waterfilling" is Player 1 optimal strategy in response to the interference induced at a given Nash equilibrium where the FDM condition holds and the individual-tone constraints are inactive.

Theorem 3. Let $\left(\hat{\mathbf{x}},\left[\hat{\mathbf{y}}^{(1)}, \ldots, \hat{\mathbf{y}}^{(n)}\right]\right)$ be a Nash equilibrium of the WCI game g. If the FDM condition holds for $g$ and $\mathbf{C}_{n}^{x} \geq$ $P^{x}$ for all $n$, then the Nash equilibrium strategy of Player 1 (namely, $\hat{\mathbf{x}}$ ) is given by "waterfilling" against the combined noise and interference $\alpha_{n} \mathbf{h}^{(n)} \hat{\mathbf{y}}^{(n)}+\mathbf{N}_{n}$ from Player 2.

Proof. Let $\left(\widehat{\mathbf{x}},\left[\hat{\mathbf{y}}^{(1)}, \ldots, \widehat{\mathbf{y}}^{(n)}\right]\right)$ be any saddle point of $J$. The condition $\mathbf{C}_{n}^{x} \geq 1 P^{x}$ ensures that the per-tone constraints are trivially satisfied whenever the power constraint $\left(P^{x}\right)$ is. Evaluating the right-hand side of (11), if $\beta_{n}=0$ (from FDM assumption), then

$$
R^{*}=\max _{\mathbf{x} \in \&_{1}} \sum_{n=1}^{N} \log \left(1+\frac{\hat{\mathbf{x}}_{n}}{\alpha_{n} \mathbf{h}^{(n)} \hat{\mathbf{y}}^{(n)}+\mathbf{N}_{n}}\right) .
$$

The optimization problem (12) is seen to be precisely the same as single-user rate maximization with parallel Gaussian channels [23], and hence the (modified) waterfilling spectrum is optimal and unique (for fixed $\left[\widehat{\mathbf{y}}^{(1)}, \ldots, \hat{\mathbf{y}}^{(n)}\right]$ ). In particular, the modified AWGN noise level on tone $n$ is seen to be $\alpha_{n} \mathbf{h}^{(n)} \hat{\mathbf{y}}^{(n)}+\mathbf{N}_{n}$. This is the same modified noise level used in the rate-adaptive IW algorithm [9].

Considering the structure of the general WCI game $g$, it is possible to establish uniqueness of Player 1 optimal strategy and strong properties of Player 2 optimal strategy. Henceforth, the set of all Nash equilibria of $q$ is denoted by $P$.

Theorem 4. The Nash equilibrium strategy of Player 1 is unique; that is, there exists some $\hat{\mathbf{x}} \in \delta_{1}$ such that for each $\left(\overline{\mathbf{x}},\left[\overline{\mathbf{y}}^{(1)}, \ldots, \overline{\mathbf{y}}^{(N)}\right]\right) \in P$, it is the case that $\overline{\mathbf{x}}=\hat{\mathbf{x}}$. Moreover, for Player 2, the induced "active" interference at each Nash equilibria is unique; in particular, $\left(\overline{\mathbf{x}},\left[\overline{\mathbf{y}}^{(1)}, \ldots\right.\right.$, $\left.\left.\overline{\mathbf{y}}^{(N)}\right]\right),\left(\widetilde{\mathbf{x}},\left[\widetilde{\mathbf{y}}^{(1)}, \ldots, \widetilde{\mathbf{y}}^{(N)}\right]\right) \in P$ imply that $\alpha_{n} \mathbf{h}^{(n)} \overline{\mathbf{y}}^{(n)}=$ $\alpha_{n} \mathbf{h}^{(n)} \tilde{\mathbf{y}}^{(n)}$ for each $n \in 1, \ldots, N$ satisfying $\hat{\mathbf{x}}_{n}>0$.

Proof. To show that Player 1 optimal strategy is identical for all Nash equilibria, consider the saddle points $\left(\overline{\mathbf{x}},\left[\overline{\mathbf{y}}^{(1)}, \ldots\right.\right.$, $\left.\left.\overline{\mathbf{y}}^{(N)}\right]\right) \in P$ and $\left(\widetilde{\mathbf{x}},\left[\tilde{\mathbf{y}}^{(1)}, \ldots, \tilde{\mathbf{y}}^{(N)}\right]\right) \in P$, which are not necessarily distinct. By Theorem 1 and separability over tones, the objective (3) is strictly concave in $\mathbf{x}$, and therefore has a unique maximizer [27], namely $\widetilde{\mathbf{x}}$, when one fixes $\left[\mathbf{y}^{(1)}, \ldots, \mathbf{y}^{(N)}\right]=\left[\tilde{\mathbf{y}}^{(1)}, \ldots, \widetilde{\mathbf{y}}^{(N)}\right]$. Observe that $\left(\overline{\mathbf{x}},\left[\widetilde{\mathbf{y}}^{(1)}, \ldots\right.\right.$, $\left.\left.\widetilde{\mathbf{y}}^{(N)}\right]\right) \in P$ by the exchangeability property of saddle points
[28]. Consequently, $\overline{\mathbf{x}}$ is also the unique maximizer of (3) for $\left[\mathbf{y}^{(1)}, \ldots, \mathbf{y}^{(N)}\right]=\left[\tilde{\mathbf{y}}^{(1)}, \ldots, \widetilde{\mathbf{y}}^{(N)}\right]$. This implies that $\overline{\mathbf{x}}=\widetilde{\mathbf{x}}$. Taking $\hat{\mathbf{x}}=\widetilde{\mathbf{x}}$ establishes the result.

To show the second claim, define $I=\left\{i: \hat{\mathbf{x}}_{i}>0\right\}$, where $\hat{\mathbf{x}}$ is the unique Nash equilibrium strategy of Player 1 as per the first claim, and suppose that there exists a nonempty set $D=\left\{n \in I: \alpha_{n} \mathbf{h}^{(n)} \overline{\mathbf{y}}^{(n)} \neq \alpha_{n} \mathbf{h}^{(n)} \tilde{\mathbf{y}}^{(n)}\right\}$. Consider $\left(\widehat{\mathbf{x}},\left[\overline{\mathbf{y}}^{(1)}, \ldots, \overline{\mathbf{y}}^{(N)}\right]\right) \in P$ and $\left(\widetilde{\mathbf{x}},\left[\widetilde{\mathbf{y}}^{(1)}, \ldots, \widetilde{\mathbf{y}}^{(N)}\right]\right) \in$ $P$, where $\widetilde{\mathbf{x}}=\overline{\mathbf{x}}=\hat{\mathbf{x}}$. Define $s_{2} \ni\left[\hat{\mathbf{y}}^{(1)}, \ldots, \hat{\mathbf{y}}^{(N)}\right]=$ $(1 / 2)\left[\overline{\mathbf{y}}^{(1)}, \ldots, \overline{\mathbf{y}}^{(N)}\right]+(1 / 2)\left[\tilde{\mathbf{y}}^{(1)}, \ldots, \tilde{\mathbf{y}}^{(N)}\right]$. The function $g$ : $\mathbb{R}_{+}^{N} \mapsto \mathbb{R}_{+}$defined by

$$
g\left(\left[\mathbf{i}_{1}, \ldots, \mathbf{i}_{N}\right]\right)=\sum_{n=1}^{N} \log \left(1+\frac{\hat{\mathbf{x}}_{n}}{\mathbf{i}_{n}+\beta_{n} \hat{\mathbf{x}}_{n}+\mathbf{N}_{n}}\right)
$$

is convex in each variable $\mathbf{i}_{n}$ and strictly convex in each variable $\mathbf{i}_{n}$ for which $n \in I$ due to (10). By the fact that $\varnothing \neq D \subset I$ and the convexity properties, it follows that $g\left(\left[\alpha_{n} \mathbf{h}^{(1)} \hat{\mathbf{y}}^{(1)}, \ldots, \alpha_{n} \mathbf{h}^{(N)} \hat{\mathbf{y}}^{(N)}\right]\right)<(1 / 2) g\left(\left[\alpha_{n} \mathbf{h}^{(1)} \overline{\mathbf{y}}^{(1)}, \ldots\right.\right.$, $\left.\left.\alpha_{n} \mathbf{h}^{(N)} \overline{\mathbf{y}}^{(N)}\right]\right)+(1 / 2) g\left(\left[\alpha_{n} \mathbf{h}^{(1)} \widetilde{\mathbf{y}}^{(1)}, \ldots, \alpha_{n} \mathbf{h}^{(N)} \tilde{\mathbf{y}}^{(N)}\right]\right)$, and consequently that

$$
\begin{aligned}
J\left(\widehat{\mathbf{x}},\left[\alpha_{n} \mathbf{h}^{(1)} \hat{\mathbf{y}}^{(1)}, \ldots, \alpha_{n} \mathbf{h}^{(N)} \hat{\mathbf{y}}^{(N)}\right]\right) \\
<\frac{1}{2} J\left(\widehat{\mathbf{x}},\left[\alpha_{n} \mathbf{h}^{(1)} \overline{\mathbf{y}}^{(1)}, \ldots, \alpha_{n} \mathbf{h}^{(N)} \overline{\mathbf{y}}^{(N)}\right]\right) \\
\left.\quad+\frac{1}{2} J\left(\hat{\mathbf{x}},\left[\alpha_{n} \mathbf{h}^{(1)} \tilde{\mathbf{y}}^{(1)}, \ldots, \alpha_{n} \mathbf{h}^{(N)}\right)^{(N)}\right]\right)=R^{*},
\end{aligned}
$$

which contradicts (5). Therefore $D=\varnothing$.

As a corollary, Theorem 4 implies that the "interference profile" $\alpha_{n} \mathbf{h}^{(n)} \mathbf{y}^{(n)}+\beta_{n} \mathbf{x}_{n}+\mathbf{N}_{n}$ is invariant on each active tone $\left\{n:\left(\mathbf{x}_{n}>0\right)\right\}$ at every Nash equilibrium. Even though the Nash equilibrium need not be unique, one therefore has a strong sense in which to speak of a worst-case interference profile that is most deleterious to Player 1. It is possible to strengthen Theorem 4 by restricting attention to the FDM setting: in Theorem 5, it is shown that in this case the structure of $P$ is polyhedral. Moreover, once one has obtained a single Nash equilibrium point, the set of all Nash equilibria may be readily deduced. This implies that the set of worstinterference profiles may be explicitly computed by practitioners for use in offline system design or dynamic operation.

Theorem 5. If the FDM condition is satisfied, then the set $P$ of all Nash equilibria of the WCI game $g$ is a polytope. ${ }^{4}$

Proof. The result is proven by constructing a polytope, $Q$ and subsequently showing that $P=Q$. To construct $Q$, take any $\left(\hat{\mathbf{x}},\left[\hat{\mathbf{y}}^{(1)}, \ldots, \hat{\mathbf{y}}^{(N)}\right]\right) \in P$ (such a point must exist by Theorem 2). Define $D=\left\{n: \widehat{\mathbf{x}}_{n}=0\right\}, E=\left\{n: 0<\widehat{\mathbf{x}}_{n}<\mathbf{C}_{n}^{x}\right\}$, $F=\left\{n: \widehat{\mathbf{x}}_{n}=\mathbf{C}_{n}^{x}\right\}$, and $I=E \cup F$. Equation (4) holds that

\footnotetext{
${ }^{4}$ Different definitions of polytopes exist in the literature; this paper defines a polytope as the bounded intersection of a finite number of half-spaces [27].
} 
$\widehat{\mathbf{x}}$ must be an optimum solution of the convex optimization problem:

$$
\begin{gathered}
\max _{\mathbf{x}} \sum_{n=1}^{N} \log \left(1+\frac{\mathbf{x}_{n}}{\alpha_{n} \mathbf{h}^{(n)} \hat{\mathbf{y}}^{(n)}+\mathbf{N}_{n}}\right), \\
\text { subject to } \mathbf{x} \geq 0, \quad n=1, \ldots, N, \\
\sum_{n} \mathbf{x}_{n} \leq P^{x}, \\
\mathbf{C}^{x} \succeq \mathbf{x} .
\end{gathered}
$$

Associate Lagrangian dual variables $\lambda \in \mathbb{R}$ and $v \in \mathbb{R}^{N}$ with constraints (17) and (18), respectively. Because the objective is concave in $\mathbf{x}$ and Slater's constraint qualification condition is satisfied [27], the Karush-Kuhn-Tucker (KKT) conditions are necessary and sufficient for optimality (for fixed $\left.\left[\mathbf{y}^{(1)}, \ldots, \mathbf{y}^{(N)}\right]=\left[\hat{\mathbf{y}}^{(1)}, \ldots, \hat{\mathbf{y}}^{(N)}\right]\right)$ :

$$
\begin{gathered}
\frac{1}{\alpha_{n} \mathbf{h}^{(n)} \mathbf{y}^{(n)}+\mathbf{x}_{n}+\mathbf{N}_{n}}-\lambda \leq 0, \quad v_{n}=0 \quad \text { if } \mathbf{x}_{n}=0, \\
\frac{1}{\alpha_{n} \mathbf{h}^{(n)} \mathbf{y}^{(n)}+\mathbf{x}_{n}+\mathbf{N}_{n}}-\lambda=0, \quad v_{n}=0 \quad \text { if } 0<\mathbf{x}_{n}<\mathbf{C}_{n}^{x}, \\
\frac{1}{\alpha_{n} \mathbf{h}^{(n)} \mathbf{y}^{(n)}+\mathbf{x}_{n}+\mathbf{N}_{n}}-\lambda-v_{n}=0, \quad \text { if } \mathbf{x}_{n}=\mathbf{C}_{n}^{x}, \\
\lambda\left(\sum_{n} \mathbf{x}_{n}-P^{x}\right)=0, \quad \mathbf{x} \in \wp_{1}, \lambda \geq 0, \nu \geq 0 .
\end{gathered}
$$

Suppose that the KKT conditions are satisfied by the triplet $\left(\widehat{\mathbf{x}}, \widehat{\lambda^{0}}, \widehat{\nu^{0}}\right)$. The triplet $\left(\widehat{\mathbf{x}}, \widehat{\lambda^{0}}, \hat{\nu}^{0}\right)$ need not be unique, in general. However, the first element is unique (by Theorem 4), and thus it remains to be seen whether the ordered pair $\left(\widehat{\lambda^{0}}, \widehat{\nu^{0}}\right)$ is unique. If $E \neq \varnothing$, then the pair is unique. To see this, consider $n_{0} \in E$ which by (20) uniquely determines $\widehat{\lambda^{0}}$ and along with (19) and (21) uniquely determines $\hat{\nu^{0}}$. Because $1 /\left(\alpha_{n_{0}} \mathbf{h}^{\left(n_{0}\right)} \hat{\mathbf{y}}^{\left(n_{0}\right)}+\mathbf{x}_{n_{0}}+\mathbf{N}_{n_{0}}\right)>0$ for all $\mathbf{x} \in \varsigma_{1}$, in account of (20) it must be that $\widehat{\lambda^{0}}>0$. In this case, we define $\hat{\lambda}=\widehat{\lambda^{0}}$ and $\hat{v}=\widehat{\nu}^{0}$.

In the event that $E=\varnothing$, observe that because the objective (15) is strictly increasing in $\mathbf{x}$, it must be that $I \neq \varnothing$. Also, because $E \subset E \cup F=I \neq \varnothing$, one has $F \neq \varnothing$. Define

$$
\begin{gathered}
\hat{\lambda}=\widehat{\lambda^{0}}+\min _{m \in F} \widehat{\nu_{m}^{0},} \\
\widehat{v_{n}}= \begin{cases}\widehat{\nu_{n}^{0}}-\min _{m \in F} \widehat{v_{m}^{0}}, & n \in F, \\
0 & \text { else. }\end{cases}
\end{gathered}
$$

It may be readily verified that $(\hat{\mathbf{x}}, \hat{\lambda}, \hat{v})$ also satisfies the KKT conditions. Observe that by (24), $\widehat{v_{n}}=0$ for at least one $n \in$ I. Because $1 /\left(\alpha_{n} \mathbf{h}^{(n)} \hat{\mathbf{y}}^{(n)}+\mathbf{x}_{n}+\mathbf{N}_{n}\right)>0$ for all $n \in I \neq \varnothing$, $\mathbf{x} \in \wp_{1},(21)$ implies that $\hat{\lambda}>0$. It is therefore the case that the triplet $(\hat{\mathbf{x}}, \hat{\lambda}, \hat{v})$ satisfies the KKT conditions and $\hat{\lambda}>0$ whether $E=\varnothing$ or $E \neq \varnothing$.
For each $n \in D$, define $\varphi_{n}$ as the solution of the equation $1 /\left(\hat{\mathbf{x}}_{n}+\varphi\right)=\hat{\lambda}+\hat{v}_{n}$, namely $\varphi_{n}=1 / \hat{\lambda}-\widehat{\mathbf{x}}_{n}$. Define the polytope

$$
\begin{aligned}
Q=\{ & \left(\mathbf{x},\left[\mathbf{y}^{(1)}, \ldots, \mathbf{y}^{(N)}\right]\right) \in \S: \mathbf{x}=\hat{\mathbf{x}}, \\
& \alpha_{n} \mathbf{h}^{(n)} \mathbf{y}^{(n)}=\alpha_{n} \mathbf{h}^{(n)} \widehat{\mathbf{y}}^{(n)} \forall n \in I, \\
& \left.\alpha_{n} \mathbf{h}^{(n)} \mathbf{y}^{(n)}+\mathbf{N}_{n} \geq \varphi_{n} \forall n \in D\right\} .
\end{aligned}
$$

It remains to be shown that $P=Q$; it is first argued that $Q \subset P$. Recall that $\left(\hat{\mathbf{x}},\left[\hat{\mathbf{y}}^{(1)}, \ldots, \hat{\mathbf{y}}^{(N)}\right]\right) \in P$ was used to construct $Q$, and consider any $\left(\tilde{\mathbf{x}},\left[\tilde{\mathbf{y}}^{(1)}, \ldots, \tilde{\mathbf{y}}^{(N)}\right]\right) \in Q$. Note that $\tilde{\mathbf{x}}=\hat{\mathbf{x}}$ by construction of $Q$. The inequality (5) requires that $\left[\widetilde{\mathbf{y}}^{(1)}, \ldots, \tilde{\mathbf{y}}^{(N)}\right]$ be an optimum solution of the convex optimization problem:

$$
\begin{aligned}
& \min _{\left[\mathbf{y}^{(1)}, \ldots, \mathbf{y}^{(N)}\right]} \sum_{n=1}^{N} \log \left(1+\frac{\hat{\mathbf{x}}_{n}}{\alpha_{n} \mathbf{h}^{(n)} \mathbf{y}^{(n)}+\beta_{n} \widehat{\mathbf{x}}_{n}+\mathbf{N}_{n}}\right) \\
& \text { subject to }\left[\mathbf{y}^{(1)}, \ldots, \mathbf{y}^{(N)}\right] \in \wp_{2} \text {. }
\end{aligned}
$$

However since by Theorem 4, $\alpha_{n} \mathbf{h}^{(n)} \tilde{\mathbf{y}}^{(n)}=\alpha_{n} \mathbf{h}^{(n)} \hat{\mathbf{y}}^{(n)}$ for all $n \in I$, the objective value is equal, and hence (5) is satisfied. Equation (4) is equivalent to requiring the KKT conditions (19)-(22) to be satisfied for some ordered pair $(\lambda, v)$, where $\mathbf{x}=\hat{\mathbf{x}}$ and $\left[\mathbf{y}^{(1)}, \ldots, \mathbf{y}^{(N)}\right]=\left[\widetilde{\mathbf{y}}^{(1)}, \ldots, \widetilde{\mathbf{y}}^{(N)}\right]$ are fixed. It is now argued that the choice of $(\lambda, \nu)=(\hat{\lambda}, \hat{\nu})$ satisfies the conditions. For each $n \in\{1, \ldots, N\}$, if $n \in D$, then $\alpha_{n} \mathbf{h}^{(n)} \tilde{\mathbf{y}}^{(n)}+\mathbf{N}_{n} \geq \varphi_{n}$ implies that $\left(\widehat{\mathbf{x}}_{n}+\alpha_{n} \mathbf{h}^{(n)} \tilde{\mathbf{y}}^{(n)}+\mathbf{N}_{n}\right)^{-1}-$ $\hat{\lambda} \leq 0$ by monotonicity of $1 /(x+a)$ in $x \geq 0$ for $a>0$. If $n \in I$, then $\alpha_{n} \mathbf{h}^{(n)} \hat{\mathbf{y}}^{(n)}+\mathbf{N}_{n}=\alpha_{n} \mathbf{h}^{(n)} \tilde{\mathbf{y}}^{(n)}+\mathbf{N}_{n}$ by construction of $Q$, and accordingly (20) or (21) is satisfied. Because both (4) and (5) are satisfied, it follows by definition that $\left(\widetilde{\mathbf{x}},\left[\widetilde{\mathbf{y}}^{(1)}, \ldots, \widetilde{\mathbf{y}}^{(N)}\right]\right) \in P$, and hence $Q \subset P$.

It is now argued that $P \subset Q$. Recall that $\left(\hat{\mathbf{x}},\left[\hat{\mathbf{y}}^{(1)}, \ldots\right.\right.$, $\left.\left.\hat{\mathbf{y}}^{(N)}\right]\right) \in P$ was used to construct $Q$ and consider any $\left(\widetilde{\mathbf{x}},\left[\tilde{\mathbf{y}}^{(1)}, \ldots, \widetilde{\mathbf{y}}^{(N)}\right]\right) \in P$. By Theorem $4, \hat{\mathbf{x}}=\widetilde{\mathbf{x}}$. Also by Theorem 4, one has $\alpha_{n} \mathbf{h}^{(n)} \widetilde{\mathbf{y}}^{(n)}=\alpha_{n} \mathbf{h}^{(n)} \hat{\mathbf{y}}^{(n)}$ for all $n \in I$, and therefore it remains only to prove that $\alpha_{n} \mathbf{h}^{(n)} \tilde{\mathbf{y}}^{(n)}+\mathbf{N}_{n} \geq \varphi_{n}$ for all $n \in D$.

Because $\left(\tilde{\mathbf{x}},\left[\tilde{\mathbf{y}}^{(1)}, \ldots, \tilde{\mathbf{y}}^{(N)}\right]\right) \in P$, there must exist a pair $\left(\widetilde{\lambda^{0}}, \tilde{\nu}^{0}\right)$ such that the triplet $\left(\widetilde{\mathbf{x}}, \widetilde{\lambda^{0}}, \widetilde{\nu^{0}}\right)$ satisfies the KKT conditions (for fixed $\left[\mathbf{y}^{(1)}, \ldots, \mathbf{y}^{(N)}\right]=\left[\widetilde{\mathbf{y}}^{(1)}, \ldots, \widetilde{\mathbf{y}}^{(N)}\right]$ ).

In the event that $E \neq \varnothing$, define $\tilde{\lambda}=\widetilde{\lambda^{0}}$ and $\tilde{\nu}=\tilde{\nu^{0}}$. Clearly, the triplet $(\tilde{\mathbf{x}}, \tilde{\lambda}, \tilde{\nu})$ also satisfies the same KKT conditions. Observe by Theorem 4 that because for $n^{\prime} \in E$ one has $\alpha_{n} \mathbf{h}^{\left(n^{\prime}\right)} \tilde{\mathbf{y}}^{\left(n^{\prime}\right)}=\alpha_{n} \mathbf{h}^{\left(n^{\prime}\right)} \hat{\mathbf{y}}^{\left(n^{\prime}\right)}$, it follows by $(20)$ that $\tilde{\lambda}=\hat{\lambda}$.

In the event that $E=\varnothing$, observe that because $\varnothing=E \subset$ $I \neq \varnothing$, we have $F=I-E \neq \varnothing$. Define

$$
\begin{gathered}
\tilde{\lambda}=\widetilde{\lambda_{n}^{0}}+\min _{m \in F} \widetilde{\nu_{m}^{0},} \\
\widetilde{\nu_{n}}= \begin{cases}\widetilde{\nu_{n}^{0}}-\min _{m \in F} \widetilde{\nu_{m}^{0}}, & n \in F, \\
0 & \text { else. }\end{cases}
\end{gathered}
$$

It may be readily verified that $(\tilde{\mathbf{x}}, \tilde{\lambda}, \tilde{\nu})$ satisfies the KKT conditions (for fixed $\left[\mathbf{y}^{(1)}, \ldots, \mathbf{y}^{(N)}\right]=\left[\tilde{\mathbf{y}}^{(1)}, \ldots, \tilde{\mathbf{y}}^{(N)}\right]$ ). By (28), there must exist some $n^{\prime} \in F$ such that $\widetilde{v_{n^{\prime}}}=0$. Similarly, recall that there must exist some $m^{\prime} \in F$ such that 
$\widehat{v_{m^{\prime}}}=0$. It is now argued that there exists some $m \in F$ such that both $\widetilde{\nu_{m}}=0$ and $\widehat{\nu_{m}}=0$. In particular, let $m=m^{\prime}$. Then by (21) and the fact that the triplet $(\hat{\mathbf{x}}, \hat{\lambda}, \hat{v})$ satisfies the KKT conditions for $\left[\mathbf{y}^{(1)}, \ldots, \mathbf{y}^{(N)}\right]=\left[\hat{\mathbf{y}}^{(1)}, \ldots, \hat{\mathbf{y}}^{(N)}\right]$, one has $1 /\left(\alpha_{m} \mathbf{h}^{(m)} \hat{\mathbf{y}}^{(m)}+\hat{\mathbf{x}}_{m}+\mathbf{N}_{m}\right) \leq 1 /\left(\alpha_{n} \mathbf{h}^{(n)} \hat{\mathbf{y}}^{(n)}+\hat{\mathbf{x}}_{n}+\mathbf{N}_{n}\right)$ for all $n \in F$. However, $\alpha_{n} \mathbf{h}^{(n)} \widetilde{\mathbf{y}}^{(n)}=\alpha_{n} \mathbf{h}^{(n)} \hat{\mathbf{y}}^{(n)}$ for all $n \in F$, and therefore $1 /\left(\alpha_{m} \mathbf{h}^{(m)} \tilde{\mathbf{y}}^{(m)}+\widetilde{\mathbf{x}}_{m}+\mathbf{N}_{m}\right) \leq 1 /\left(\alpha_{n} \mathbf{h}^{(n)} \tilde{\mathbf{y}}^{(n)}+\widetilde{\mathbf{x}}_{n}+\mathbf{N}_{n}\right)$ for all $n \in F$. This (along with the fact that $\widetilde{v_{n^{\prime}}}=0$ for some $n^{\prime} \in F$ ) implies that $\widetilde{\nu_{m}}=0$. Then, (21) for this choice of $m$ implies that $\tilde{\lambda}=\hat{\lambda}$.

Because it is always the case that $\hat{\lambda}=\tilde{\lambda}$, the triplet $(\hat{\mathbf{x}}, \hat{\lambda}, \tilde{v})$ satisfies the KKT conditions (for $\left[\mathbf{y}^{(1)}, \ldots, \mathbf{y}^{(N)}\right]=$ $\left.\left[\tilde{\mathbf{y}}^{(1)}, \ldots, \tilde{\mathbf{y}}^{(N)}\right]\right)$. Therefore, $1 /\left(\alpha_{n} \mathbf{h}^{(n)} \tilde{\mathbf{y}}^{(n)}+\widehat{\mathbf{x}}_{n}+\mathbf{N}_{n}\right)-\hat{\lambda} \leq 0$ for all $n \in D$ implies that $\alpha_{n} \mathbf{h}^{(n)} \tilde{\mathbf{y}}^{(n)}+\mathbf{N}_{n} \geq \varphi_{n}$ for all $n \in D$. Thus $\left(\tilde{\mathbf{x}},\left[\tilde{\mathbf{y}}^{(1)}, \ldots, \tilde{\mathbf{y}}^{(N)}\right]\right) \in Q$.

\subsection{Numerical computation of the saddle point}

In order to apply the WCI bound in practical settings, it is necessary to develop numerical algorithms to solve for Nash equilibrium strategies and $R^{*}$. The methodology considered herein is that of interior-point optimization techniques such as the "infeasible start Newton method" [27, Section 10.3]. The general approach of interior-point techniques is to replace the (power and positivity) constraints with barrier functions that become large as the (power and positivity) constraints become tight. By making the increase in the barrier functions progressively sharper, one solves a sequence of problems whose solutions converge to a Nash equilibrium of $g$. We now formally cast the problem (11) in the interior-point setting and argue that it satisfies certain necessary properties needed for convergence. Logarithmic barrier functions are employed to enforce the positivity and power constraints and a Newton-step central path algorithm is used to compute $R^{*}$ to arbitrary accuracy [27].

Let the central path parameter be denoted by $t \in \mathbb{R}_{++}$ and define $\widetilde{\vartheta_{1}}=\operatorname{int}\left(\wp_{1}\right), \widetilde{\vartheta_{2}}=\operatorname{int}\left(\wp_{2}\right)$, and $\tilde{J}: \widetilde{\vartheta_{1}} \times \widetilde{\vartheta_{2}} \mapsto \mathbb{R}_{+}$, where

$$
\begin{aligned}
& \tilde{J}\left(\mathbf{x},\left[\mathbf{y}^{(1)}, \ldots, \mathbf{y}^{(N)}\right]\right) \\
& =t^{-1} \log \left(P^{x}-\sum_{n=1}^{N} \mathbf{x}_{n}\right)+\sum_{n=1}^{N} t^{-1} \log \left(\mathbf{C}_{n}^{x}-\mathbf{x}_{n}\right) \\
& +\sum_{n=1}^{N}\left\{\log \left(1+\frac{\mathbf{x}_{n}}{\alpha_{n} \mathbf{h}^{(n)} \mathbf{y}^{(n)}+\beta_{n} \mathbf{x}_{n}+\mathbf{N}_{n}}\right)+t^{-1} \log \left(\mathbf{x}_{n}\right)\right. \\
& \left.\quad-t^{-1} \sum_{l=1}^{2 L}\left[\log \left(\mathbf{y}_{l}^{(n)}\right)+\log \left(\mathbf{C}_{l}^{y,(n)}-\mathbf{y}_{l}^{(n)}\right)\right]\right\} \\
& \quad-t^{-1} \sum_{l=1}^{2 L} \log \left(\mathbf{P}_{l}^{y}-\sum_{n=1}^{N} \mathbf{y}_{l}^{(n)}\right) .
\end{aligned}
$$

To establish convergence, it is necessary only to show that $\tilde{J}$ satisfies the following sufficient conditions [27, Section 10.3.4] that the sublevel sets of $\|\nabla \tilde{J}\|_{2}$ are closed, and that the Hessian of $\tilde{J}$ is Lipschitz continuous with bounded inverse.
The partial derivatives of $\tilde{J}$,

$$
\begin{aligned}
\frac{\partial \tilde{J}}{\partial \mathbf{x}_{n}}= & \frac{\alpha_{n} \mathbf{h}^{(n)} \mathbf{y}^{(n)}+\mathbf{N}_{n}}{\left(\beta_{n} \mathbf{x}_{n}+\alpha_{n} \mathbf{h}^{(n)} \mathbf{y}^{(n)}+\mathbf{N}_{n}\right)\left(\left(1+\beta_{n}\right) \mathbf{x}_{n} \alpha_{n} \mathbf{h}^{(n)} \mathbf{y}^{(n)}+\mathbf{N}_{n}\right)} \\
& +\frac{1}{t \mathbf{x}_{n}}-\frac{1}{t\left(P^{x}-\mathbf{1}^{T} \mathbf{x}\right)}-\frac{1}{t\left(\mathbf{C}_{n}^{x}-\mathbf{x}_{n}\right)}, \\
\frac{\partial \tilde{J}}{\partial\left(\mathbf{y}_{m}^{(n)}\right)} & \\
= & \frac{\alpha_{n} \mathbf{h}_{m}^{(n)}}{\left(1+\beta_{n}\right) \mathbf{x}_{n}+\alpha_{n} \mathbf{h}^{(n)} \mathbf{y}^{(n)}+\mathbf{N}_{n}}-\frac{\alpha_{n} \mathbf{h}_{m}^{(n)}}{\beta_{n} \mathbf{x}_{n}+\alpha_{n} \mathbf{h}^{(n)} \mathbf{y}^{(n)}+\mathbf{N}_{n}} \\
& -\frac{1}{t \mathbf{y}_{m}^{(n)}}+\frac{1}{t\left(\mathbf{C}_{m}^{y,(n)}-\mathbf{y}_{m}^{(n)}\right)}+\frac{1}{t\left(\mathbf{P}_{m}^{y}-\sum_{n=1}^{N} \mathbf{y}_{m}^{(n)}\right)},
\end{aligned}
$$

are continuous on $\tilde{\delta}_{1} \times \tilde{S}_{2}$, implying by continuity of the

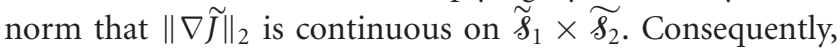
the sublevel sets $S_{\alpha}$ for each $\alpha \in \mathbb{R}$,

$$
\begin{aligned}
& S_{\alpha}=\left\{\left(\mathbf{x},\left[\mathbf{y}^{(1)}, \ldots, \mathbf{y}^{(N)}\right]\right) \in \widetilde{\Im_{1}} \times \widetilde{\Im_{2}}:\right. \\
&\left.\left\|\nabla \tilde{J}\left(\left(\mathbf{x},\left[\mathbf{y}^{(1)}, \ldots, \mathbf{y}^{(N)}\right]\right)\right)\right\|_{2} \leq \alpha\right\},
\end{aligned}
$$

are closed relative to $\widetilde{8_{1}} \times \widetilde{8_{2}}$. To show that $S_{\alpha}$ is closed, suppose that $\left\{z_{n}\right\}$ is any sequence in $S_{\alpha}$ with $z_{n} \rightarrow z$. If $z \in \widetilde{s_{1}} \times \widetilde{s_{2}}=\operatorname{int}\left(\widetilde{s_{1}} \times \widetilde{s_{2}}\right)$, then $z \in S_{\alpha}$ by relative closure. Therefore, it remains only to observe that there does not exist any $z_{n} \rightarrow z$ with $z \in \partial \operatorname{cl}\left(\widetilde{\vartheta_{1}} \times \widetilde{\oiint_{2}}\right)$. This follows from examining (30), where it can be seen that $\left\|\nabla \tilde{J}\left(z_{n}\right)\right\|_{2}$ increases without bound for any such $z_{n} \rightarrow z$. This contradicts the assumption that $\left\{z_{n}\right\}$ is a sequence in $S_{\alpha}$.

In order to show for arbitrary $\alpha \in \mathbb{R}$ that the Hessian is Lipschitz continuous on $S_{\alpha}$, it is enough to show that each element of $\nabla^{2} \tilde{J}$ is continuously differentiable on $S_{\alpha}$. The partial derivatives of (30) may be readily computed ${ }^{5}$ and seen to be continuous functions on $S_{\alpha} \subset \delta_{1} \times \delta_{2}$. However, $\ell_{1} \times s_{2}$ is bounded, therefore $S_{\alpha}$ is also bounded (and closed), hence compact. Therefore, each partial derivative of $\nabla^{2} \widetilde{J}$, as a continuous function on a compact set, is bounded. Finally, the bounded inverse condition on the Hessian follows from the fact that the barrier functions are strictly concave in $\mathbf{x}$ and strictly convex in $\left[\mathbf{y}^{(1)}, \ldots, \mathbf{y}^{(N)}\right]$. In particular, computation of the Hessian reveals that $\nabla_{\mathbf{x}}^{2} \tilde{J} \preceq\left(-t^{-1} /\left(P^{x}\right)^{2}\right) I$ and $\nabla_{\left[\mathbf{y}^{(1)}, \ldots, \mathbf{y}^{(N)}\right]}^{2} \succeq\left(t^{-1} / \max _{i}\left(\mathbf{P}^{y}\right)_{i}^{2}\right) I$ on $\widetilde{\delta_{1}} \times{\widetilde{\vartheta_{2}}}_{2}$, and hence $S_{\alpha}$.

\section{SIMULATION RESULTS}

The scope of the WCI analysis extends generally to DMTbased DSL systems. This section examines two particular cases that are deployed prevalently: VDSL and ADSL. In VDSL, a prominent interference issue is the upstream

\footnotetext{
5 The expressions are lengthy and omitted for space.
} 


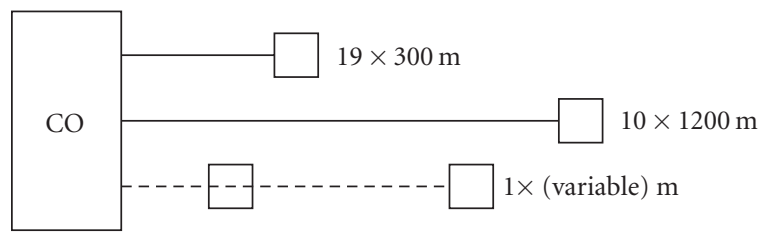

FIgURE 2: Binder configuration for upstream VDSL simulations (not to scale). The dashed line is of varying lengths.

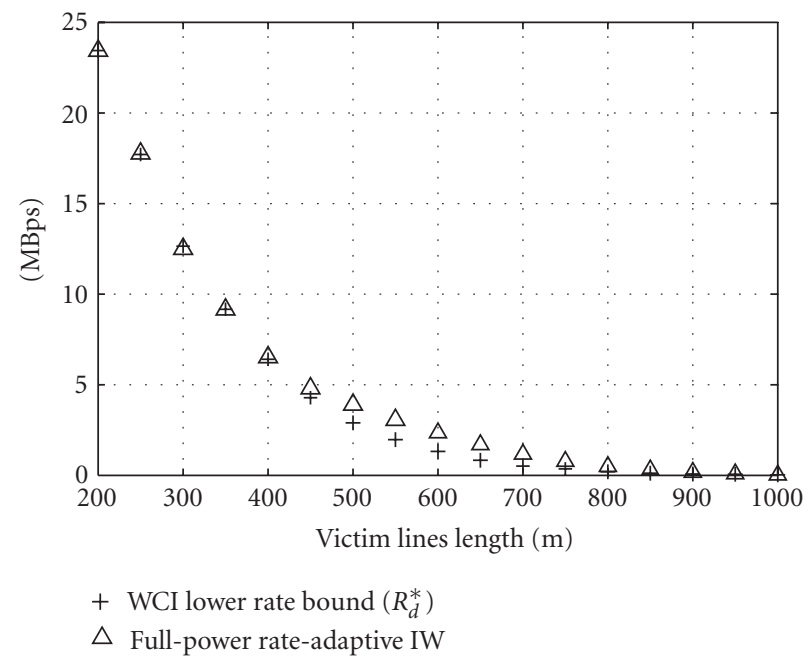

FIgURE 3: Achievable rates in upstream VDSL as a function of victim lines length (200-1000 m).

near-far effect, which is caused by crosstalk from short("near") lines FEXT coupling into longer ("far") lines. In ADSL, the issue of RT FEXT injection into longer CO lines is similarly of concern. Numerical results for these sample deployments demonstrate the practicality of the WCI analysis and show surprising commonalities between the different scenarios. In all simulations, the interior-point technique is used with an error tolerance of less than $0.1 \%$.

\subsection{VDSL upstream}

The WCI rate bound is first applied to two different upstream VDSL scenarios exhibiting the near-far effect. The binder configuration is illustrated in Figure 2. For all simulations, $19 \times 300 \mathrm{~m}$ lines, $10 \times 1200 \mathrm{~m}$ lines, and one line of varying length occupy the binder of 24 AWG twistedpairs. The FTTEx M2 (998 FDM) bandplan is employed with HAM bands notched and the usual PSD constraints removed. Tones below $138 \mathrm{kHz}$ are disabled for ADSL compatibility, and the normal PSD masks are not applied. The FDM condition is satisfied for this configuration, hence $\beta_{n}=0$. For $10^{-7} \mathrm{BER}$, assume coding gain of $3 \mathrm{~dB}$, with $6 \mathrm{~dB}$ margin, thus $\Gamma=12.5 \mathrm{~dB}$. Each line is limited to $14.5 \mathrm{dBm}$ power $\left(P^{x}=14.5 \mathrm{dBm}, \mathbf{P}^{y}=\mathbf{1} \cdot 14.5 \mathrm{dBm}\right)$.

\subsubsection{WCI rate as a function of line length}

First, consider the WCI rate bound when the variable-length line is the victim line (Player 1). Numerical results are shown in Figure 3, where a lower bound rate as well as the rate obtained when all lines execute full-power rate-adaptive (RA) IW are plotted as a function of victim line length. Note that full-power RA IW is quite different from fixed-margin (FM) IW, where power is minimized while achieving a fixed rate and margin [18]. To investigate practical bit loading constraints numerically, RA IW with discrete bit constraints [9] is executed on the victim modem assuming the WCI (11). Player 1 achieved rate with discrete bit loading is plotted as $R_{d}^{*}$. Evidently, $R_{d}^{*} \leq R^{*}$, and therefore $R_{d}^{*}$ is also a lower bound to the achievable rate under the WCI.

Observe that for most line lengths, the rate achieved by RA IW is fairly close to the WCI bound, particularly near $200 \mathrm{~m}$ and $900 \mathrm{~m}$. For intermediate lengths $(\approx 650 \mathrm{~m})$, rateadaptive IW can perform up to $\approx 75 \%$ better than the WCI bound, though the absolute difference is small. As a corollary, the interference generated by IW in this configuration is deleterious in the sense that it is close in rate to the WCI saddle point. This finding is consistent with results [11] showing that other centralized DSM strategies can significantly outperform IW in such cases. Furthermore, fixed-margin (FM) IW can also be seen to perform significantly better than the WCI bound when rates are adjudicated reasonably [18].

\subsubsection{WCI rate as a function of $P B O$}

Motivated by the results of the previous section showing that the full-power WCI rate bound can decrease precipitously as loop length increases, the efficacy of upstream power backoff (UPBO) at mitigating this effect is considered. This section examines a simple power-backoff strategy in the form of power-constrained RA IW for Level 0-1 DSM. Though the use of RA IW is retained, an effect similar to fixed-margin (rate-constrained) IW [18] is induced by imposing various tighter sum power constraints. In particular, the variablelength line is set to length $300 \mathrm{~m}$, and (sum) power backoff is imposed on all (20) $300 \mathrm{~m}$ lines with full power retained on the (10) $1200 \mathrm{~m}$ lines. By taking the victim line to be one of the $300 \mathrm{~m}$ lines, the $300 \mathrm{~m}$ WCI curve in Figure 4 is generated, yielding a lower bound to the achievable data rate for all $300 \mathrm{~m}$ lines in the binder. The $1200 \mathrm{~m}$ WCI curve represents the case where the victim modem is instead taken to be one of the $1200 \mathrm{~m}$ lines. To compare standardized SSM techniques to DSM, the rates achieved using the SSM VDSL 


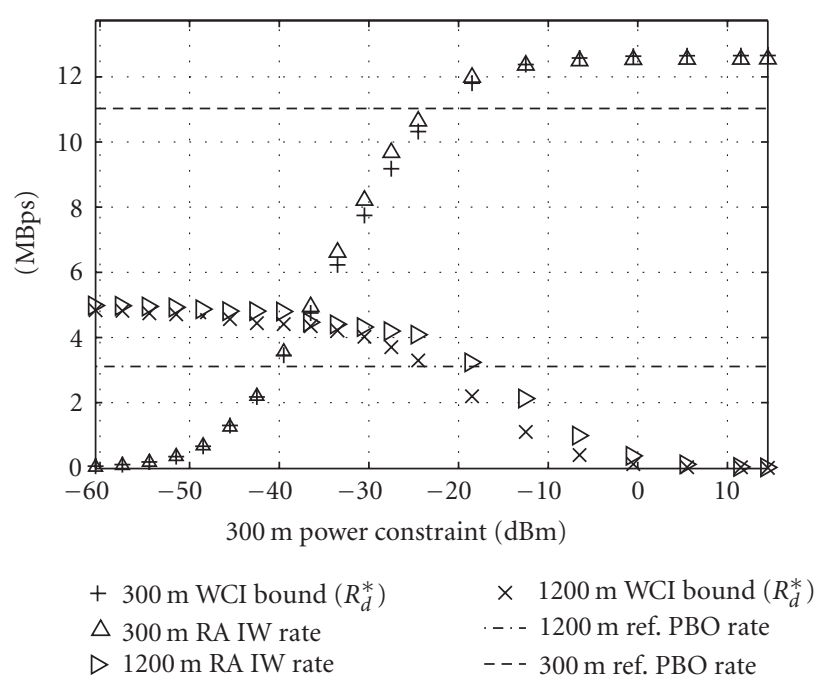

FIGURE 4: Achievable rates in upstream VDSL as a function of shortline $(300 \mathrm{~m})$ power backoff.

UBPO masking technique defined for the noise A environment [29] are illustrated by dashed horizontal lines.

The results illustrate that a tradeoff exists between the rates of the short and long lines. Examining the $1200 \mathrm{~m}$ lines, the proposed technique improves both the RA IWachieved and WCI bounds significantly up to approximately $-30 \mathrm{dBm}$, with diminishing returns for further PBO as the $300 \mathrm{~m}$ line FEXT no longer dominates the interference profile. However, further PBO decreases the achievable rates of the $300 \mathrm{~m}$ lines, as expected. The WCI bound is again fairly tight. Thus by employing such a simple PBO scheme with Level 1 DSM, one can dynamically control the tradeoff between short and long lines to best match desired operating conditions, that is, operating with guaranteed $\approx 4 \mathrm{MBps}$ on the $1200 \mathrm{~m}$ lines and $\approx 7.75 \mathrm{MBps}$ on the $300 \mathrm{~m}$ lines. In this example, the SSM technique achieves approximately the same performance as this simple DSM technique at one tradeoff point $(\approx-22 \mathrm{~dB} \mathrm{PBO})$.

\subsection{ADSL downstream with remote terminals (RTs)}

The WCI rate bound is also applicable to ADSL. This section considers an RT ADSL configuration as illustrated in Figure 5. For all simulations, 25 ADSL lines are located $2000 \mathrm{~m}$ from a fiber-fed RT $4000 \mathrm{~m}$ from the CO. Additionally, $5 \times 5000 \mathrm{~m}$ lines are present in the binder. The FDM ADSL standard [30] parameters are assumed. As in the VDSL simulations, $\Gamma=12.5 \mathrm{~dB}$. Each line is limited to $20.4 \mathrm{dBm}$ downstream power $\left(P^{x}=20.4 \mathrm{dBm}, \mathbf{P}^{y}=\mathbf{1} \cdot 20.4 \mathrm{dBm}\right)$, and the standard PSD masks are neglected.

A common problem of such configurations is that the signal from the CO to the non-RT $(7000 \mathrm{~m})$ modems will be saturated by FEXT from the RT lines. As in the VDSL example, the efficacy of (sum) power backoff for the RT lines as a means of improving the rate of the CO lines is studied. Figure 6 shows the dependence of rates on the level of

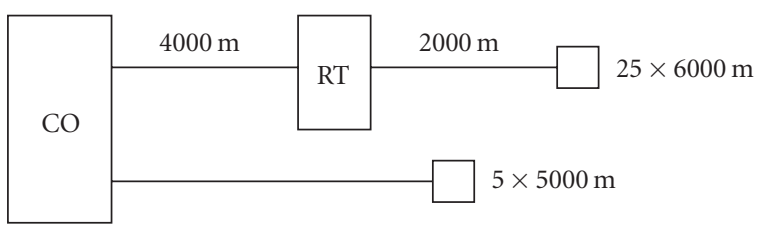

Figure 5: Binder configuration for downstream RT ADSL simulations (not to scale). A common RT is used for each line.

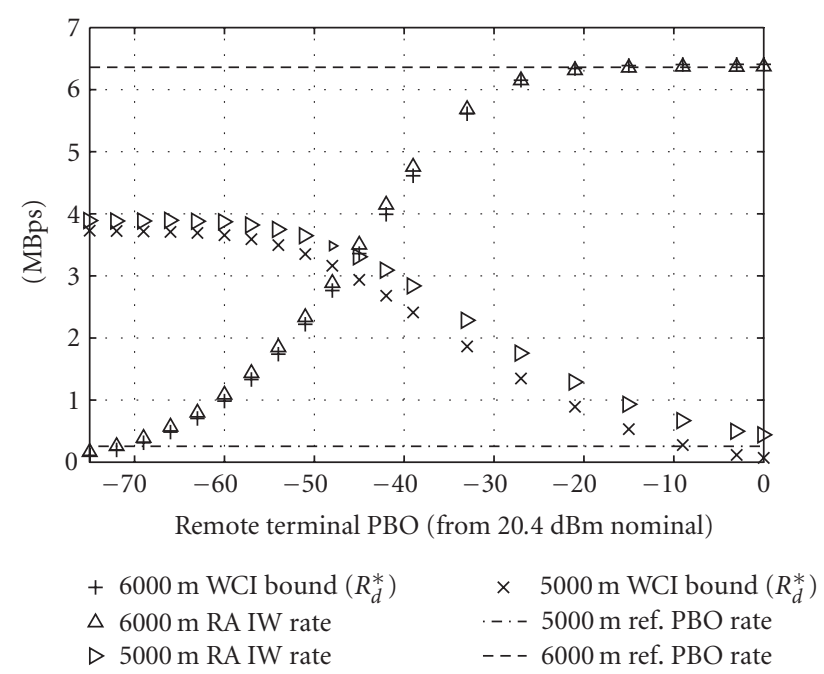

Figure 6: Achievable rates in downstream ADSL as a function of RT line power backoff (relative to $20.4 \mathrm{dBm}$ nominal TX power).

power backoff (relative to $20.4 \mathrm{dBm}$ ) for the RT lines. The horizontal lines represent the performance obtained by SSM with the standardized PSD masks.

The WCI bound is reasonably close to actual powercontrolled RA IW performance on both RT and CO lines. Figure 7 shows the spectrum adopted at the (approximate) Nash equilibrium, as well as the power allocation chosen by discrete IW against the noise induced by Player 2, yielding $R_{d}^{*}$ (in discrete IW, tones above 47 are not used because they correspond to fractional bit loadings). The simulation shows that Player 1 interference is dominated by interference from the RT modems; these modems induce a "kindred-like" noise while the $\mathrm{CO}$ lines concentrate their power at low frequencies. Also illustrated by example is that the Player 2 optimal strategy may be highly frequency-selective, and therefore the existing interference analysis technique of setting tight PSD masks for each modem cannot capture the WCI unless the masks are set very high. ${ }^{6}$ As in VDSL, a wide range of useful operating points may be attained; for example, it is possible (through proper power control) to guarantee $3 \mathrm{MBps}$ service on all lines, whereas this rate point was far from being feasible with SSM or with full-power rate-adaptive IW. However

\footnotetext{
${ }^{6}$ Doing so would consistently overestimate interference power, and underestimate achievable DSM performance.
} 


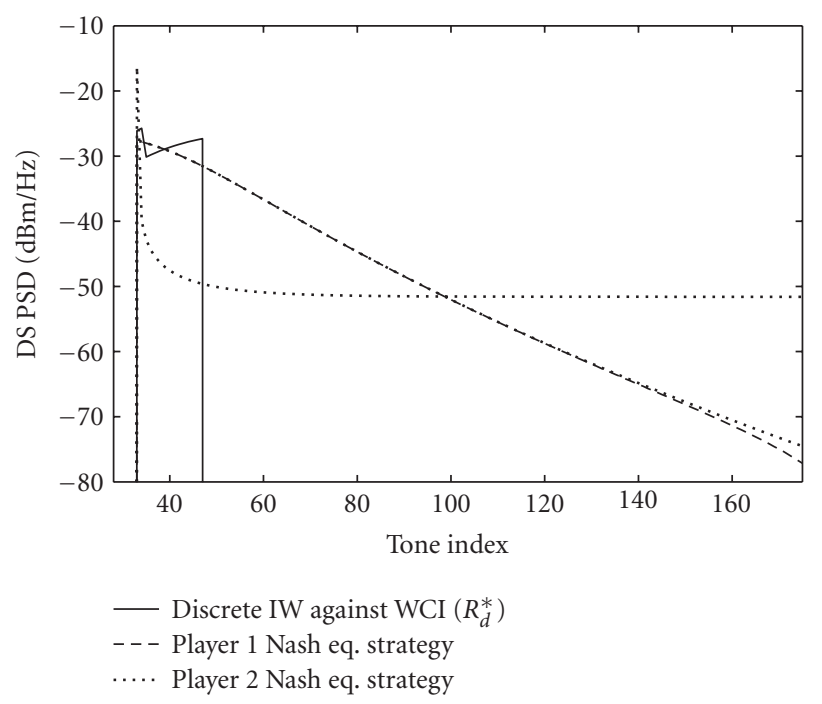

FIgURE 7: Spectral allocations $\left(\mathbf{x},\left[\mathbf{y}^{(1)}, \ldots, \mathbf{y}^{(N)}\right]\right)$ of players 1 and 2 for the rightmost lower $(0 \mathrm{~dB} \mathrm{PBO})$ operating point in Figure 6, where player 1 is a CO line. Note that the RT line spectrum overlaps $\mathbf{x}$ on most tones.

without any power backoff, the performance of RA IW and the WCI bound is near that of SSM, showing the key role of power control in obtaining DSM gains in this setting.

\section{CONCLUSION}

This paper has studied the worst-case interference encountered when deploying Level 0-2 DSM techniques for nextgeneration DSL. A game-theoretic analysis has shown that under mild conditions, a pure-strategy Nash equilibrium exists in the WCI game, and can be computed using standard optimization techniques. The Nash equilibrium provides a useful lower bound to the achievable rate for a DSL modem employing DSM under any power-constrained interference profile. Furthermore, the structure of the Nash equilibrium reveals that for FDM systems, IW is optimal in a maximin sense.

The WCI bound was applied to a Level 0-1 upstream near-far VDSL scenario and was found to be numerically tight. The utility of a simple DSM UPBO strategy employing RA IW was compared to SSM UPBO, were it was found that control of rate tradeoffs is possible with DSM, which may allow significantly preferable operating rates. A similar tradeoff was observed in RT ADSL systems, where CO line performance benefits significantly from proper power control. These results suggest that the parameter of transmit power is important to DSM performance, in the sense that proper power control can beget large performance gains in this setting.

\section{ACKNOWLEDGMENT}

The research was supported by NSF under Contract CNS0427677 and by the Stanford Graduate Fellowship Program.

\section{REFERENCES}

[1] "Spectrum management for loop transmission systems," ANSI Std. T1.417, 2002.

[2] I. Sason, "On achievable rate regions for the Gaussian interference channel," IEEE Transactions on Information Theory, vol. 50, no. 6, pp. 1345-1356, 2004.

[3] W. C. Peng, Some communication jamming games, Ph.D. thesis, University of Southern California, Los Angeles, Calif, USA, 1986.

[4] M. V. Hegde, W. E. Stark, and D. Teneketzis, "On the capacity of channels with unknown interference," IEEE Transactions on Information Theory, vol. 35, no. 4, pp. 770-783, 1989.

[5] T. Han and K. Kobayashi, "A new achievable rate region for the interference channel," IEEE Transactions on Information Theory, vol. 27, no. 1, pp. 49-60, 1981.

[6] S. T. Chung and J. M. Cioffi, "The capacity region of frequency-selective Gaussian interference channels under strong interference," in Proceedings of IEEE International Conference on Communications (ICC '03), vol. 4, pp. 2753-2757, Anchorage, Alaska, USA, May 2003.

[7] K. B. Song, S. T. Chung, G. Ginis, and J. M. Cioffi, "Dynamic spectrum management for next-generation DSL systems," IEEE Communications Magazine, vol. 40, no. 10, pp. 101-109, 2002.

[8] K. J. Kerpez, D. L. Waring, S. Galli, J. Dixon, and P. Madon, "Advanced DSL management," IEEE Communications Magazine, vol. 41, no. 9, pp. 116-123, 2003.

[9] T. Starr, M. Sorbara, J. M. Cioffi, and P. J. Silverman, DSL Advances, Prentice-Hall PTR, Upper Saddle River, NJ, USA, 2003.

[10] S. T. Chung, S. J. Kim, J. Lee, and J. M. Cioffi, "A gametheoretic approach to power allocation in frequency-selective Gaussian interference channels," in Proceedings of IEEE International Symposium on Information Theory (ISIT '03), pp. 316-316, Pacifico Yokohama, Kanagawa, Japan, June-July 2003.

[11] R. Cendrillon, M. Moonen, J. Verliden, T. Bostoen, and W. $\mathrm{Yu}$, "Optimal multiuser spectrum management for digital subscriber lines," in Proceedings of IEEE International Conference on Communications (ICC '04), vol. 1, pp. 1-5, Paris, France, June 2004.

[12] D. Statovci and T. Nordstrom, "Adaptive subcarrier allocation, power control, and power allocation for multiuser FDD-DMT systems," in Proceedings of IEEE International Conference on Communications (ICC '04), vol. 1, pp. 11-15, Paris, France, June 2004.

[13] G. Cherubini, "Optimum upstream power back-off and multiuser detection for VDSL," in Proceedings of IEEE Global Telecommunications Conference (GLOBECOM '01), vol. 1, pp. 375-380, San Antonio, Tex, USA, November 2001.

[14] J. Lee, R. V. Sonalkar, and J. M. Cioffi, "Multi-user discrete bit-loading for DMT-based DSL systems," in Proceedings of IEEE Global Telecommunications Conference (GLOBECOM '02), vol. 2, pp. 1259-1263, Taipei, Taiwan, November 2002.

[15] K. S. Jacobsen, "Methods of upstream power backoff on very high speed digital subscriber lines," IEEE Communications Magazine, vol. 39, no. 3, pp. 210-216, 2001.

[16] S. Schelstraete, "Defining upstream power backoff for VDSL," IEEE Journal on Selected Areas in Communications, vol. 20, no. 5, pp. 1064-1074, 2002.

[17] K.-M. Kang and G.-H. Im, "Upstream power back-off method for VDSL transmission systems," IEE Electronics Letters, vol. 39, no. 7, pp. 634-635, 2003. 
[18] W. Yu, G. Ginis, and J. M. Cioffi, "Distributed multiuser power control for digital subscriber lines," IEEE Journal on Selected Areas in Communications, vol. 20, no. 5, pp. 1105-1115, 2002.

[19] M. Ho, J. M. Cioffi, and J. A. C. Bingham, "Discrete multitone echo cancelation," IEEE Transactions on Communications, vol. 44, no. 7, pp. 817-825, 1996.

[20] K. Van Acker, M. Moonen, and T. Pollet, "Per-tone echo cancellation for DMT-based systems," IEEE Transactions on Communications, vol. 51, no. 9, pp. 1582-1590, 2003.

[21] G. Ysebaert, K. Vanbleu, G. Cuypers, M. Moonen, and J. Verlinden, "Echo cancellation for discrete multitone frameasynchronous ADSL transceivers," in Proceedings of IEEE International Conference on Communications (ICC '03), vol. 4, pp. 2421-2425, Anchorage, Alaska, USA, May 2003.

[22] D. C. Jones, "Frequency domain echo cancellation for discrete multitone asymmetric digital subscriber line transceivers," IEEE Transactions on Communications, vol. 43, no. 2-4, pp. 1663-1672, 1995.

[23] T. M. Cover and J. A. Thomas, Elements of Information Theory, John Wiley \& Sons, New York, NY, USA, 1991.

[24] S. Schelestrate ed., "Very high speed digital subscriber lines, part 3: Multicarrier modulation (MCM) specification," ANSI Std. T1.424, 2002.

[25] B. Widrow and S. D. Streams, Adaptive Signal Processing, Prentice-Hall, Englewood Cliffs, NJ, USA, 1985.

[26] J. M. Cioffi, "Incentive-based spectrum management," T1.E1 Contribution 2004/480R2, August 2004.

[27] S. Boyd and L. Vandenberghe, Convex Optimization, Cambridge University Press, Cambridge, UK, 2004.

[28] T. Basar and G. J. Olsder, Dynamic Noncooperative Game Theory, Academic Press, New York, NY, USA, 1982.

[29] "Very high speed digital subscriber lines, part 1: Metallic interface," ANSI T1.424 (Draft), February 2004.

[30] ITR Recommendations G.992.1, "Asymmetric digital subscriber line (ADSL) transceivers," ITU, June 1999.

Mark H. Brady received his B.S.E.E degree in 2001 from the University of Illinois at Urbana-Champaign, and his M.S.E.E degree from Stanford University in 2003. He is presently a Doctoral candidate at Stanford University under the supervision of Professor John Cioffi. His research interests include DSL systems, optimization theory, and information theory.

John M. Cioffi received his B.S.E.E. degree in 1978 from University of Illinois and he received his Ph.D.E.E. degree in 1984 from Stanford University. He was with Bell Laboratories from 1978 to 1984 and with IBM Research from 1984 to 1986. He has been a Professor of electrical engineering at Stanford University since 1986. He founded Amati Com. Corp. in 1991 (purchased by TI in 1997) and was Officer/Director from 1991 to 1997. He currently is on the Board of Directors of Marvell, ASSIA, Inc. (Chair), Teranetics, and ClariPhy. $\mathrm{He}$ is on the Advisory Board of Portview Ventures and Wavion. His specific interests are in the area of high-performance digital transmission. He is the holder of Hitachi America Professorship in Electrical Engineering at Stanford (2002); he is a Member
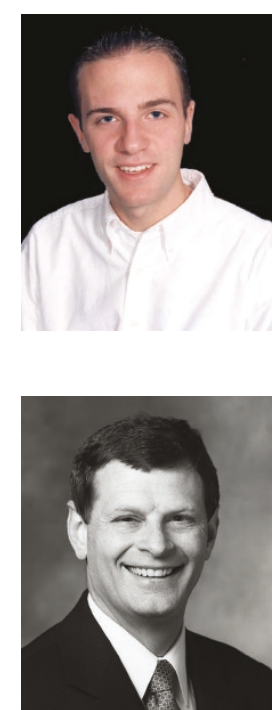

of the National Academy of Engineering (2001); IEEE Kobayashi Medal (2001); IEEE Millennium Medal (2000); IEEE Fellow (1996); IEE J.J. Tomson Medal (2000); 1999 University of Illinois Outstanding Alumnus, 1991 IEEE Communications Magazine Best Paper; 1995 ANSI T1 Outstanding Achievement Award; NSF Presidential Investigator (1987-1992), ISSLS 2004 Outstanding Paper Award. He has published over 250 papers and holds over 40 patents. 